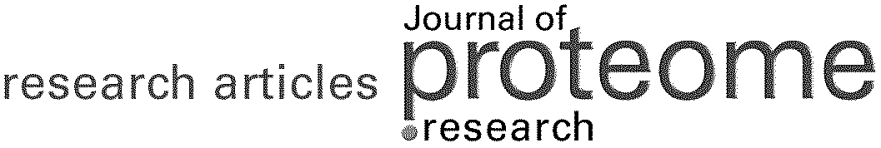

\section{Proteomic Analysis of Calcium Oxalate Monohydrate Crystal-Induced Cytotoxicity in Distal Renal Tubular Cells}

\author{
Visith Thongboonkerd, ${ }^{*, \dagger}$ Theptida Semangoen, ${ }^{\dagger, \ddagger}$ Supachok Sinchaikul, ${ }^{\S}$ and Shui-Tein Chen $^{\S, \|}$ \\ Medical Proteomics Unit \& Medical Molecular Biology Unit, Office for Research and Development, Faculty of \\ Medicine Siriraj Hospital, Mahidol University, Bangkok, Thailand, Department of Immunology and \\ Immunology Graduate Program, Faculty of Medicine Siriraj Hospital, Mahidol University, Bangkok, Thailand, \\ Institute of Biological Chemistry and Genomic Research Center, Academia Sinica, Taipei, Taiwan, and Institute \\ of Biochemical Sciences, College of Life Science, National Taiwan University, Taipei, Taiwan
}

Received April 1, 2008

\begin{abstract}
Calcium oxalate monohydrate (COM) is the major crystalline component found in kidney stones and its adhesion to renal tubular cells provokes tubular injury, which in turn enhances COM crystal adhesion. However, COM-induced toxic effects in these tubular cells remain largely unknown. We performed a proteomics study to characterize changes in the cellular proteome in MDCK distal renal tubular cells after an exposure to high-dose $(1000 \mu \mathrm{g} / \mathrm{mL})$ COM crystals for $48 \mathrm{~h}$, at which percentage of cell death was significantly increased. Proteins were extracted from MDCK cells cultured with COM-containing or COM-free medium ( $n=5$ individual flasks per group), resolved in individual 2-D gels, and stained with SYPRO Ruby fluorescence dye. Quantitative and statistical analyses revealed 53 proteins whose abundance levels were altered ( 25 were increased, whereas other 28 were decreased) by COM-induced toxicity. Among these, 50 were successfully identified by quadrupole time-of-flight (Q-TOF) mass spectrometry (MS) and/or tandem MS (MS/MS) analyses. The proteomic data were clearly confirmed by 2-D Western blot analysis. While three chaperones (GRP78, Orp150 and Hsp60) were increased, other proteins involved in protein biosynthesis, ATP synthesis, cell cycle regulator, cellular structure, and signal transduction were decreased. These data provide some novel mechanistic insights into the molecular mechanisms of COM crystal-induced tubular toxicity.
\end{abstract}

Keywords: Calcium oxalate $\bullet$ Cellular responses $\bullet$ Crystal adhesion • Kidney • Proteome • Proteomics - Stone - Tubular toxicity

\section{Introduction}

Kidney stone disease (nephrolithiasis) remains a common health problem worldwide. ${ }^{1,2}$ Among all types of kidney stones, calcium oxalate monohydrate (COM) is the major crystalline compound found in the stone mass (with a frequency of up to $77.5 \%) .{ }^{3}$ In addition to crystallization, crystal growth and crystal aggregation, the other crucial mechanism for COM kidney stone formation is adhesion of COM crystals to renal tubular epithelial cells. ${ }^{4,5}$ Adhesion of COM crystals can induce injury and apoptosis of renal tubular epithelial cells, and vice versa, COM-induced cellular injury can facilitate COM crystal adhesion. ${ }^{6-9}$ This vicious cycle therefore accelerates kidney stone formation. Understanding alterations in renal tubular

* To whom correspondence should be addressed. Visith Thongboonkerd, M.D., FRCPT, Medical Proteomics Unit, Office for Research and Development, 12th Floor Adulyadej Vikrom Building, 2 Prannok Road, Siriraj Hospital, Bangkoknoi, Bangkok 10700, Thailand. Phone/Fax: +66-2-4184973. E-mail: thongboonkerd@dr.com or vthongbo@yahoo.com

${ }^{\dagger}$ Medical Proteomics Unit \& Medical Molecular Biology Unit, Mahidol University.

${ }^{\ddagger}$ Department of Immunology and Immunology Graduate Program, Mahidol University.

${ }^{\S}$ Institute of Biological Chemistry and Genomic Research Center, Academia Sinica.

"Institute of Biochemical Sciences, National Taiwan University. cells induced by COM crystals may lead to an identification of molecular targets for the prevention of kidney stone formation. However, changes in renal tubular epithelial cells during COM crystal-induced toxicity remain largely unknown. Several previous studies, which had dissected this vicious cycle, unfortunately focused mainly on redox or oxidative stress pathways, ${ }^{9-11}$ particularly in mitochondria. ${ }^{12,13}$ Clearer image of global changes in COM crystal-induced renal tubular toxicity is thus required.

During the postgenomic era, proteomics has become one of the most promising tools in nephrology and urology fields to unravel pathogenic mechanisms underlying renal and urological diseases. ${ }^{14-16}$ The advantage of the proteomics approach is that both the previously determined and undetermined proteins that are involved in the disease mechanisms can be simultaneously examined. In the present study, we employed a classical proteomics approach to identify changes in the cellular proteome as a response to COM crystal-induced toxicity in MDCK distal renal tubular cells. The rationale of using MDCK cell line was that it exhibits several features of tubular cells in the distal nephron, ${ }^{17}$ which has been thought to be the primary site of kidney stone formation. Moreover, MDCK cell line has been frequently used in several of previous 


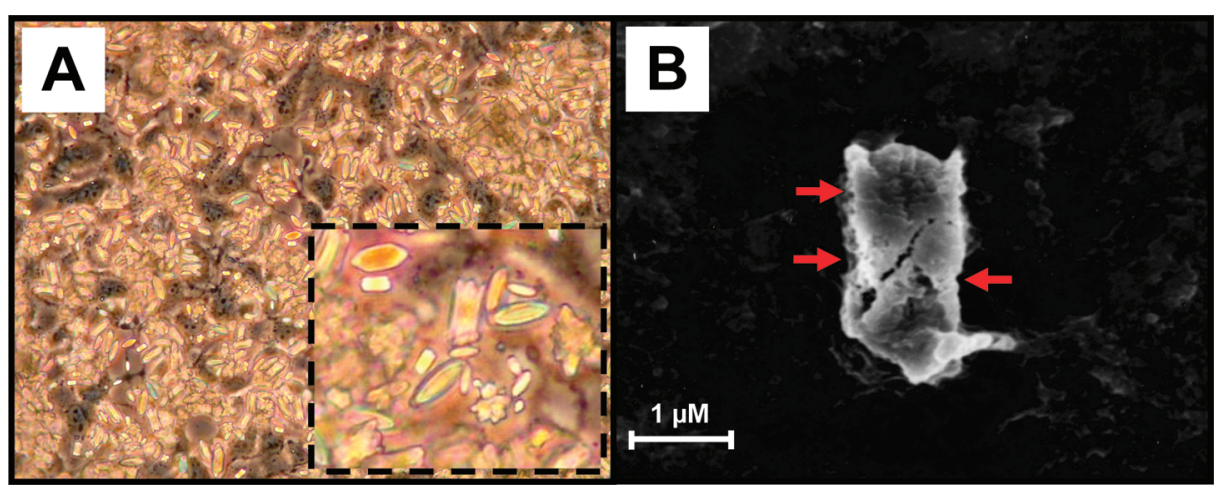

Figure 1. COM crystal adhesion and cell-crystal interactions. Images were taken using phase-contrast microscopy (A) and scanning electron microscopy (SEM) (B) after an incubation of COM crystals $(1000 \mu \mathrm{g} / \mathrm{mL})$ with MDCK cells for $48 \mathrm{~h}$. An inset in (A) is a zoom-in phase-contrast microscopic image demonstrating the COM crystals adhered on the MDCK cell monolayer. SEM image in (B) shows blurred or interrupted borders (illustrated with arrows) of COM crystals (which indicate cell-crystal interactions) with the background of the monolayer of MDCK cells. Original magnification power was $100 \times$ in (A) and $2000 \times$ in (B).

studies on COM crystal-induced renal tubular cell injury. ${ }^{18} \mathrm{~A}$ high-dose $(1000 \mu \mathrm{g} / \mathrm{mL})$ of COM crystals used in the present study caused significantly increased cell death at 48-h after the exposure to the crystals. Two-dimensional electrophoresis (2DE) followed by quadrupole time-of-flight (Q-TOF) mass spectrometry (MS) and/or tandem MS (MS/MS) analyses revealed significant changes in abundance levels of several proteins in these COM-induced toxic MDCK cells compared to controls. The functional roles and subcellular localizations of these altered proteins indicated that these changes were related, at least in part, to COM crystal-induced tubular injury and ultimately to kidney stone formation.

\section{Materials and Methods}

Preparation of COM Crystals. COM crystals were prepared as previously described. ${ }^{19}$ Briefly, $10 \mathrm{mM}$ calcium chloride dihydrate $\left(\mathrm{CaCl}_{2} \cdot 2 \mathrm{H}_{2} \mathrm{O}\right)$ was mixed with $10 \mathrm{mM}$ sodium oxalate $\left(\mathrm{Na}_{2} \mathrm{C}_{2} \mathrm{O}_{4}\right)$ to make final concentrations of $5 \mathrm{mM}$ and $0.5 \mathrm{mM}$, respectively, in Tris buffer containing $90 \mathrm{mM} \mathrm{NaCl}(\mathrm{pH} 7.4$ ). The mixture was incubated at $25{ }^{\circ} \mathrm{C}$ overnight and COM crystals were harvested by centrifugation at $3000 \mathrm{rpm}$ for $5 \mathrm{~min}$. Supernatant was discarded and the crystals were resuspended in methanol. After another centrifugation at $3000 \mathrm{rpm}$ for 5 min, methanol was discarded and the crystals were dried at $37{ }^{\circ} \mathrm{C}$ overnight. COM crystals were then decontaminated by UV light radiation for $30 \mathrm{~min}$. They were then added to a complete Eagle's minimum essential medium (MEM) (GIBCO, Invitrogen Corporation; Grand Island, NY) to achieve the final concentration of $1000 \mu \mathrm{g} / \mathrm{mL}$. These in vitro COM crystals had similar size and shape as those of in vivo samples found in the urine of kidney stone patients.

Cell Culture and COM Crystal Adhesion. Approximately 3 $\times 10^{6}$ MDCK cells were inoculated in each $75 \mathrm{~cm}^{2}$ tissue culture flask containing MEM supplemented with $10 \%$ fetal bovine serum (FBS), $1.2 \%$ penicillinG/streptomycin and $2 \mathrm{mM}$ glutamine. The cultured cells were maintained in a humidified incubator at $37^{\circ} \mathrm{C}$ with $5 \% \mathrm{CO}_{2}$ for $24 \mathrm{~h}$. A total of 10 semiconfluent flasks were then divided into two groups ( $n=5$ per group) and the culture medium was replaced by either COM-containing (with $1000 \mu \mathrm{g} / \mathrm{mL}$ COM crystals) or COM-free medium. For the COMfree medium, COM crystals with an equal amount of $1000 \mu \mathrm{g} /$
$\mathrm{mL}$ were added into the medium for $30 \mathrm{~min}$, but were finally removed from the medium by centrifugation at $3000 \mathrm{rpm}$ for $5 \mathrm{~min}$.

Imaging by Phase-Contrast Microscopy and Scanning Electron Microscopy (SEM). Phase-contrast microscopy was simply performed on an inverted light microscope (Olympus CKX41, Olympus Co. Ltd.; Tokyo, Japan) after three washes (with PBS) of the MDCK cells incubated with COM crystals $(1000 \mu \mathrm{g} / \mathrm{mL})$ in $75 \mathrm{~cm}^{2}$ tissue culture flasks, as mentioned above. For the evaluation by SEM, the cell culture and COM crystal adhesion were performed exactly the same as aforementioned, but on coverslips, instead of culture flasks (with approximately $1 \times 10^{6}$ cells per slide at an initial inoculation). After incubation with COM crystals $(1000 \mu \mathrm{g} / \mathrm{mL})$, the coverslips were rinsed with normal saline solution (NSS) three times and fixed with $2 \%$ glutaraldehyde in NSS at room temperature for $2 \mathrm{~h}$. The coverslips were then rinsed again with NSS three times and dehydrated by a graded ethanol series of $50 \%, 70 \%, 95 \%$ and $100 \%$ before being air-dried overnight. The coverslips were finally mounted on aluminum stubs and coated with gold particles. The crystal morphology and adhesion were then examined under a scanning electron microscope (JSM-25S, Jeol; Kyoto, Japan).

Cell Death Assay. Apoptosis was detected by the determination of surface phosphatidylserine, which was translocated from the inner side of plasma membranes to outer layer of the cell during apoptotic cell death. After trypsinization, FITClabeled annexin V, a calcium-dependent phospholipid-binding protein with a high affinity for phosphatidylserine, was added to discriminate healthy from apoptotic cells. Additionally, propidium iodide, a DNA stain, was used simultaneously to detect necrotic cells. MDCK cells from the monolayer were detached with $0.1 \%$ trypsin in 2.5 mM EDTA and resuspended in $10 \mathrm{~mL}$ of MEM. The harvested cells were centrifuged at 1500 $\mathrm{rpm}, 4^{\circ} \mathrm{C}$ for $5 \mathrm{~min}$, and washed with PBS. Cell pellets were resuspended with annexin $\mathrm{V}$ buffer (10 mM HEPES, $140 \mathrm{mM}$ $\mathrm{NaCl}$ and $2.5 \mathrm{mM} \mathrm{CaCl}_{2} \cdot 2 \mathrm{H}_{2} \mathrm{O} ; \mathrm{pH} 7.4$ ) at a final concentration of $5 \times 10^{5}$ cells $/ \mathrm{mL}$ and then incubated with FITC-labeled annexin V (BD Biosciences; San Jose, CA) on ice for $15 \mathrm{~min}$ in the dark. Propidium iodide (BD Biosciences) was added into the samples at a final concentration of $10 \mu \mathrm{L} / \mathrm{mL}$ prior to analysis. The cells were then analyzed by flow cytometry (FACScan, Becton Dickinson Immunocytometry System; San 
Control

$12 \mathrm{~h}$

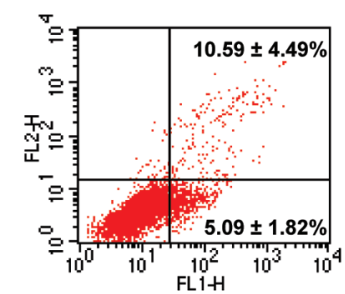

$24 \mathrm{~h}$

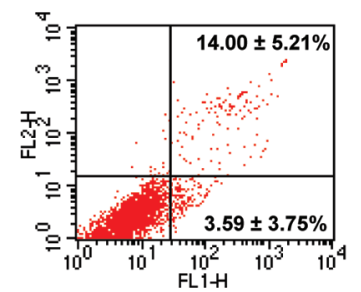

$48 \mathrm{~h}$



COM
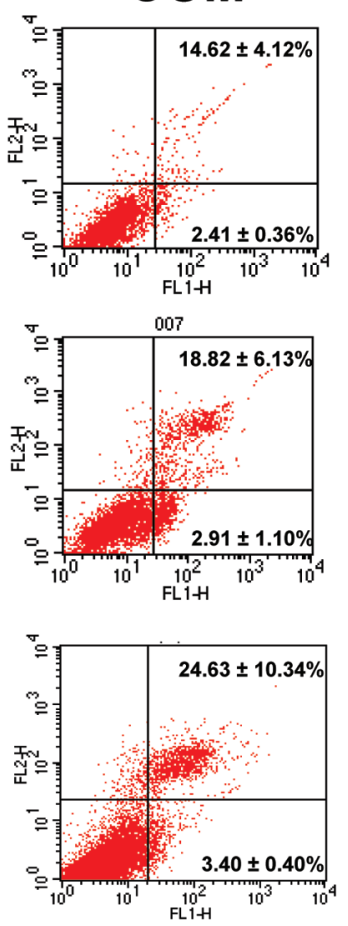

$\square$ Early apoptosis

Late apoptos is \& necrosis

- Total cell death

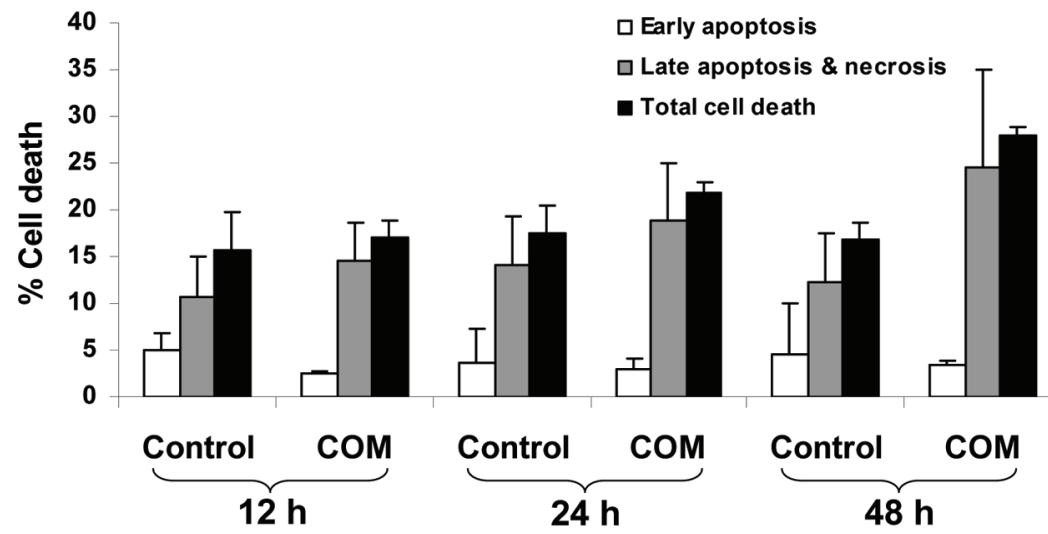

Figure 2. Flow cytometric data of cell death during exposure to COM crystals. MDCK cells were cultured without (control) or with COM crystals $(1000 \mu \mathrm{g} / \mathrm{mL})$. Apoptosis and necrosis of these cells were evaluated using annexin V (FL1-H)/propidium iodide (FL2-H) double staining. The percentage of cell death was determined by a summation of the number of cells in the right upper quadrant (late apoptotic cells and/or necrotic cells), left upper quadrant (necrotic cells), and right lower quadrant (early apoptotic cells) compared to total cell count in all four quadrants. Lower panel shows Mean (+SD) of cell death data obtained from 3 independent experiments.

Jose, CA) and a monolayer of MDCK cells treated with $2 \mu \mathrm{g} /$ $\mathrm{mL}$ camptothecin was used as a positive control. This experiment was performed in triplicates. Percentage of cell death $(\%$ cell death $)=[$ (number of both apoptotic and necrotic cells/ number of all cells) $\times 100 \%]$.

Protein Extraction. After incubation with or without COM crystals, the monolayer of MDCK cells was harvested by directly scraping into the tube containing 0.5 M EDTA in PBS to dissolve the adherent $\mathrm{CaOx}$ crystals followed by an incubation at $4{ }^{\circ} \mathrm{C}$ for $30 \mathrm{~min}$. EDTA was then removed by washing with PBS three times. After a centrifugation at $10000 \mathrm{rpm}$ for $2 \mathrm{~min}$, the cell pellet was washed with PBS three times and was then resuspended in a buffer containing $7 \mathrm{M}$ urea, $2 \mathrm{M}$ thiourea, 4\% 3-[(3cholamidopropyl) dimethyl-ammonio]-1-propanesulfonate (CHAPS), $120 \mathrm{mM}$ dithiothreitol (DTT), 2\% ampholytes (pH $3-10$ ) and $40 \mathrm{mM}$ Tris- $\mathrm{HCl}$, and further incubated at $4{ }^{\circ} \mathrm{C}$ for $30 \mathrm{~min}$. Unsolubilized debris and particulate matters were removed by centrifugation at $10000 \mathrm{rpm}$ for $2 \mathrm{~min}$. Protein concentrations were determined using the Bradford method.

2-DE and Staining. Protein solutions (each of $200 \mu \mathrm{g}$ total protein) derived from individual culture flasks ( $n=5$ for each group) were premixed with a rehydration buffer containing 7 M urea, 2 M thiourea, 2\% CHAPS, 120 mM DTT, 40 mM Trisbase, $2 \%$ ampholytes ( $\mathrm{pH} \mathrm{3-10)}$ and a trace of bromophenol blue to make the final volume of $150 \mu \mathrm{L}$ per sample. The mixtures were rehydrated onto Immobiline DryStrip (nonlinear $\mathrm{pH}$ gradient of 3-10, $7 \mathrm{~cm}$ long; GE Healthcare, Uppsala, Sweden) at room temperature for 10-15 h. The first-dimensional separation or isoelectric focusing (IEF) was performed in Ettan IPGphor II IEF System (GE Healthcare) at $20^{\circ} \mathrm{C}$, using a stepwise mode to reach $9000 \mathrm{Vh}$. After completion of the IEF, the strips were first equilibrated for $15 \mathrm{~min}$ in an equilibration buffer containing $6 \mathrm{M}$ urea, $130 \mathrm{mM}$ DTT, $112 \mathrm{mM}$ Tris-base, $4 \%$ SDS, $30 \%$ glycerol and $0.002 \%$ bromophenol blue, and then 

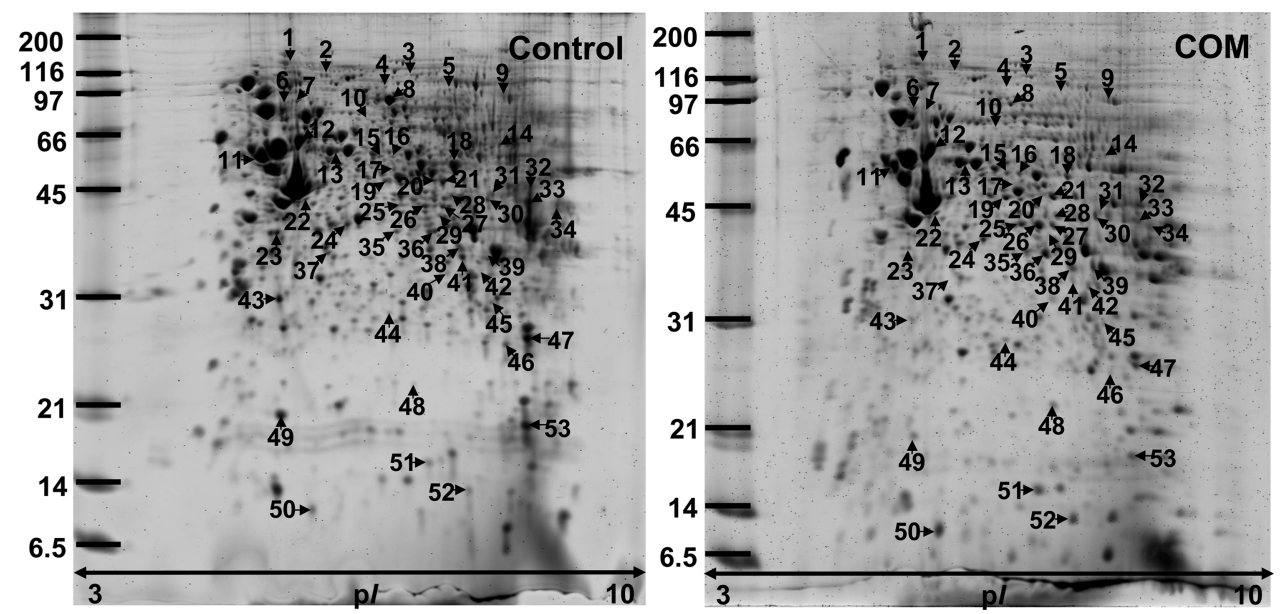

Figure 3. Representative 2-D gel images of proteins derived from the control and COM-exposed MDCK cells. Proteins were resolved with 2-DE using a pH gradient of 3-10 (nonlinear) for the IEF and 12\% polyacrylamide SDS-PAGE for the second-dimensional separation. The resolved protein spots were then visualized with SYPRO Ruby stain. Each gel contained $200 \mu \mathrm{g}$ of total protein extracted from each culture flask ( $n=5$ for each group; total $n=10$ gels were used for quantitative intensity analysis). The spots labeled with numbers are those whose intensity levels significantly differed between the two groups. These differentially expressed proteins were subsequently identified by Q-TOF MS and/or MS/MS analyses (see Table 1).

in another similar buffer, which replaced DTT with $135 \mathrm{mM}$ iodoacetamide, for further $15 \mathrm{~min}$. The second-dimensional separation or SDS-PAGE was performed in $12 \%$ polyacrylamide gel using SE260 mini-Vertical Electrophoresis Unit (GE Healthcare) at $150 \mathrm{~V}$ for approximately $2 \mathrm{~h}$. Separated proteins were visualized with SYPRO Ruby fluorescence staining (InvitrogenMolecular Probes; Eugene, OR). Gel images were taken using a Typhoon laser scanner (GE Healthcare).

Matching and Analysis of Protein Spots. Image Master 2D Platinum (GE Healthcare) software was used for matching and analysis of protein spots in 2-D gels. Parameters used for spot detection were (i) minimal area $=10$ pixels; (ii) smooth factor $=2.0$; and (iii) saliency $=2.0$. A reference gel was created from an artificial gel combining all of the (common and uncommon) spots presenting in different gels into one image. This reference gel was then used for matching the corresponding protein spots among all different gels within the same group ( $n=5$ gels per group) and between the two different groups (i.e., control vs COM-exposed cells). The spots were automatically aligned and matched using some selected landmarks, which were the welldefined spots that were present in all gels. However, each of the matched spots by this automatic option was then confirmed or edited manually. Background subtraction was performed and the intensity volume of each spot was normalized with total intensity volume (summation of the intensity volumes obtained from all spots within the same 2-D gel).

Statistical Analysis. Comparisons between the two sets of the samples (i.e., control vs COM-exposed cells) of \% cell death and intensity levels of corresponding protein spots were performed using unpaired Student's $t$ test. $P$-values less than 0.05 were considered statistically significant. Differentially expressed protein spots, which were statistically significant, were subjected to in-gel tryptic digestion and identification by mass spectrometry.

In-Gel Tryptic Digestion. The protein spots whose intensity levels significantly differed between groups were excised from 2-D gels, washed twice with $200 \mu \mathrm{L}$ of $50 \%$ acetonitrile (ACN)/ $25 \mathrm{mM} \mathrm{NH}_{4} \mathrm{HCO}_{3}$ buffer (pH 8.0) at room temperature for 15 min, and then washed once with $200 \mu \mathrm{L}$ of $100 \%$ ACN. After washing, the solvent was removed, and the gel pieces were dried by a SpeedVac concentrator (Savant; Holbrook, NY) and rehydrated with $10 \mu \mathrm{L}$ of $1 \%(\mathrm{w} / \mathrm{v})$ trypsin (Promega; Madison, WI) in $25 \mathrm{mM} \mathrm{NH}_{4} \mathrm{HCO}_{3}$. After rehydration, the gel pieces were crushed with siliconized blue stick and incubated at $37^{\circ} \mathrm{C}$ for at least $16 \mathrm{~h}$. Peptides were subsequently extracted twice with $50 \mu \mathrm{L}$ of $50 \%$ ACN/5\% trifluoroacetic acid (TFA); the extracted solutions were then combined and dried with the SpeedVac concentrator. The peptide pellets were resuspended with 10 $\mu \mathrm{L}$ of $0.1 \%$ TFA and purified using ZipTip ${ }_{\mathrm{C} 18}$ (Millipore; Bedford, MA). The peptide solution was drawn up and down in the ZipTip $\mathrm{C}_{18} 10$ times and then washed with $10 \mu \mathrm{L}$ of $0.1 \%$ formic acid by drawing up and expelling the washing solution three times. The peptides were finally eluted with $5 \mu \mathrm{L}$ of $75 \%$ ACN $/ 0.1 \%$ formic acid.

Protein Identification by MALDI-Q-TOF MS and MS/MS Analyses. The proteolytic samples were premixed 1:1 with the matrix solution $(5 \mathrm{mg} / \mathrm{mL} \alpha$-cyano-4-hydroxycinnamic acid (CHCA) in 50\% ACN, 0.1\% (v/v) TFA and 2\% (w/v) ammonium citrate) and spotted onto the 96-well sample stage. The samples were analyzed by the Q-TOF Ultima mass spectrometer (Micromass; Manchester, U.K.), which was fully automated with predefined probe motion pattern and the peak intensity threshold for switching over from MS survey scanning to MS/ MS, and from one MS/MS to another. Within each sample well, parent ions that met the predefined criteria (any peak within the $m / z$ 800-3000 range with intensity above 10 count \pm include/exclude list) were selected for CID MS/MS using argon as the collision gas and a mass dependent $\pm 5 \mathrm{~V}$ rolling collision energy until the end of the probe pattern was reached. The LM and HM resolution of the quadrupole were both set at 10 to give a precursor selection window of about 4-Da wide. Manual acquisition and optimization for individual samples or peaks was also possible.

The instrument was externally calibrated to $<5 \mathrm{ppm}$ accuracy over the mass range of $\mathrm{m} / z$ 800-3000 using a sodium iodide and PEG 200, 600, 1000 and 2000 mixtures and further adjusted with Glu-Fibrinopeptide B as the near-point lock mass calibrant during data processing. At a laser firing rate of $10 \mathrm{~Hz}$, individual spectra from 5 -s integration period acquired for each of the MS survey and MS/MS performed were combined, 


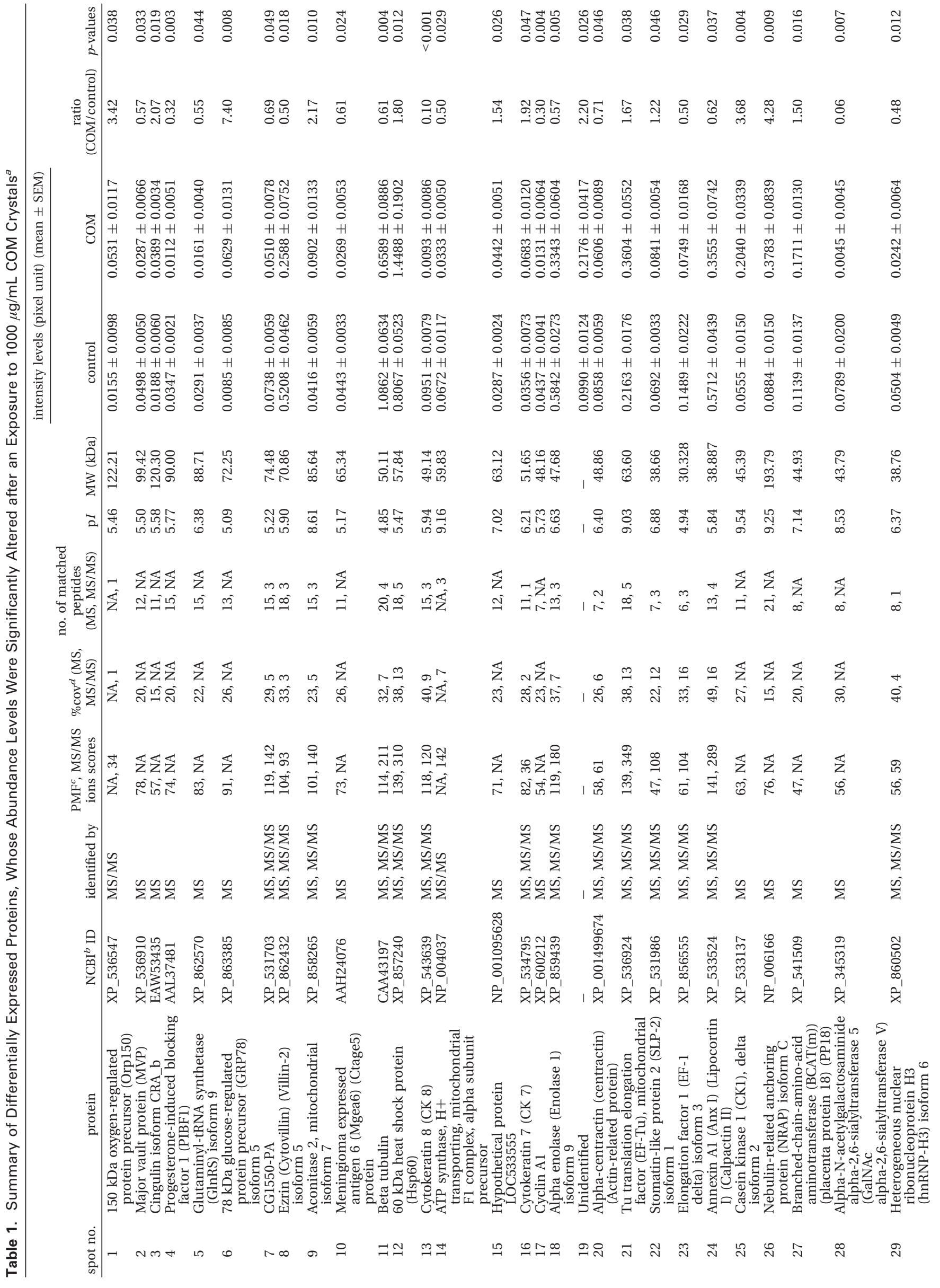




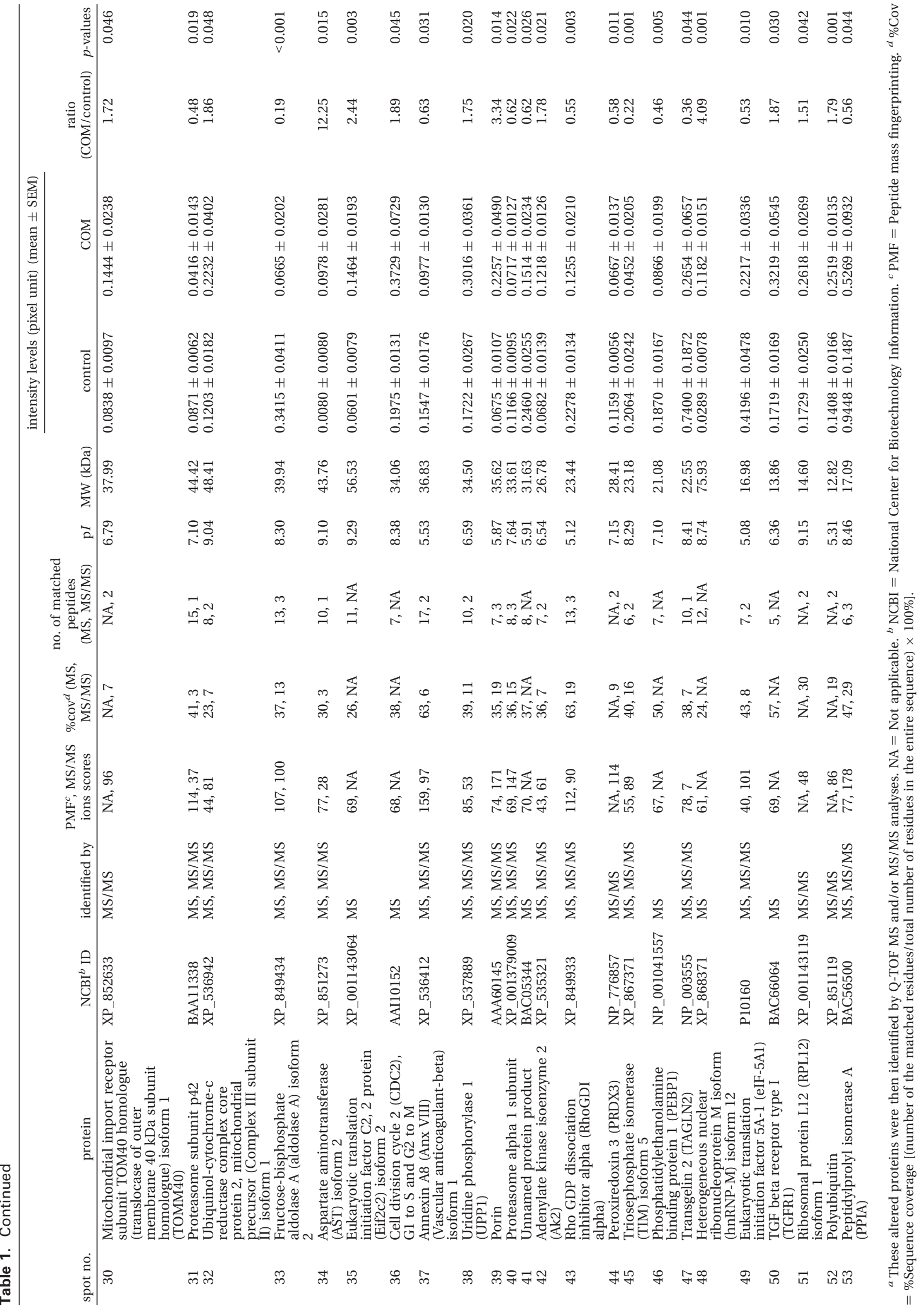



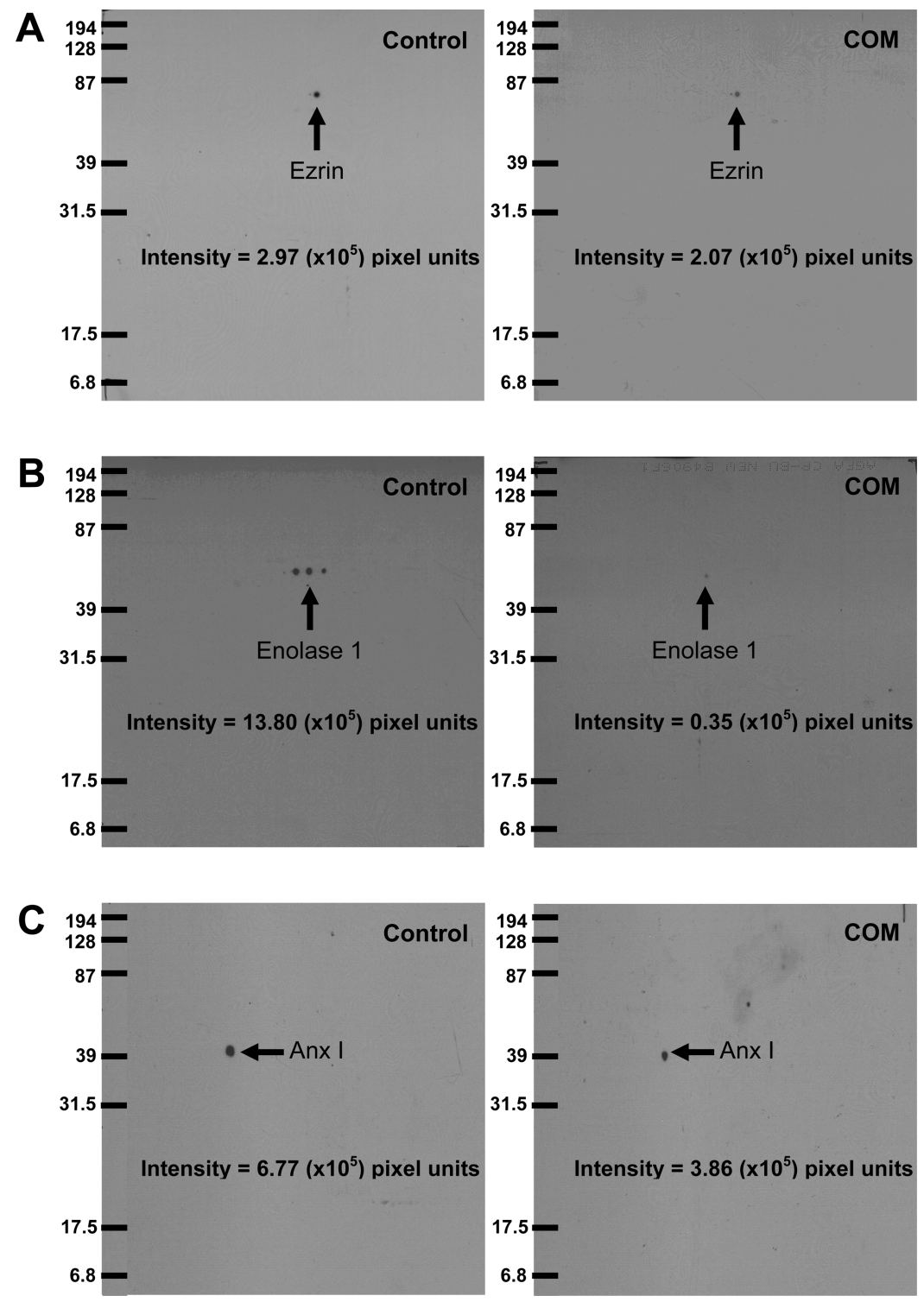

Figure 4. Validation of the proteomic data by 2-D Western blot analysis. Proteins derived from MDCK cells (100 $\mu \mathrm{g}$ of total protein for each sample; extraction was performed as for 2-DE analysis) were resolved with 2-DE and transferred onto nitrocellulose membranes. After blocking nonspecific bindings, the membranes were incubated with mouse polyclonal anti-ezrin (A), rabbit polyclonal anti-enolase 1 (B), and mouse monoclonal anti-Anx I (C) primary antibodies, and then with their respective secondary antibodies conjugated with horseradish peroxidase. Immunoreactive protein spots were then visualized with chemiluminescence substrate.

smoothed, deisotoped (fast option) and centroided using the ProteinLynx GlobalSERVER 2.0 data processing software (Micromass). The MS and MS/MS data were extracted and outputted as the searchable .txt and .pkl files, respectively, for independent searches using the public MASCOT search engine (http://www.matrixscience.com). The data were searched against the NCBI mammalian protein database (with approximately $5.7 \times 10^{6}$ entries) assuming that peptides were monoisotopic. Fixed modification was carbamidomethylation at cysteine residues, whereas variable modification was oxidation at methionine residues. Only 1 missed trypsin cleavage was allowed, and peptide mass tolerances of 100 and 50 ppm were allowed for peptide mass fingerprinting and MS/MS ions search, respectively.

2-D Western Blotting. To confirm the results of MS and/or MS/MS protein identification, 2-D Western blot analysis was performed. Proteins derived from MDCK cells (100 $\mu \mathrm{g}$ of total protein for each sample; extraction was performed as for 2-DE analysis) were resolved by 2-DE (as described above) and transferred onto nitrocellulose membranes (Whatman, Dassel, Germany) using a semidry transfer apparatus (Bio-Rad, Milano, Italy) at $75 \mathrm{~mA}$ for $1 \mathrm{~h}$. Nonspecific bindings were blocked with $5 \%$ milk in PBS at room temperature for $1 \mathrm{~h}$. The membranes were then incubated with mouse polyclonal anti-ezrin (1:500 in 5\% milk/PBS) (Santa Cruz Biotechnology, Inc., Santa Cruz, $\mathrm{CA})$, rabbit polyclonal anti-enolase 1 (1:200 in 5\% milk/PBS) (Santa Cruz Biotechnology, Inc.) and mouse monoclonal antiAnx I (1:500 in 5\% milk/PBS) (CHEMICON International, Inc., Temecula, CA) primary antibodies at $4{ }^{\circ} \mathrm{C}$ overnight. After washing, the membranes were further incubated with respective secondary antibodies conjugated with horseradish peroxidase (with dilutions of 1:2000 to 1:5000 in 5\% milk/PBS) at room temperature for $1 \mathrm{~h}$. Immunoreactive protein spots were then visualized with SuperSignal West Pico chemiluminescence substrate (Pierce Biotechnology, Inc., Rockford, IL). 
Table 2. Functional Roles and Subcellular Localizations of the Altered Proteins Identified from the COM-Exposed MDCK Cells ${ }^{a}$

\begin{tabular}{|c|c|c|}
\hline altered proteins & subcellular localization & function \\
\hline \multicolumn{3}{|c|}{ Proteins Whose Abundance Levels Were Significantly Increased during COM Crystal Adhesion } \\
\hline Aconitase2, mitochondrial isoform 7 & Mitochondria & Metabolic enzyme \\
\hline Ak 2 isoform 1 & Mitochondrial intermembrane space & Metabolic enzyme \\
\hline $\mathrm{BCAT}(\mathrm{m})$ & Mitochondria & Metabolic enzyme \\
\hline $\mathrm{CDC} 2$ & Nucleus & Metabolic enzyme \\
\hline Cingulin isoform CRA_b & Tight junction & Cellular structure \\
\hline CKldelta isoform 2 & Cytosol-Golgi and ER-Golgi transport vesicles & Vesicular trafficking \\
\hline Eif $2 \mathrm{c} 2$ isoform 2 & Cytoplasm & Protein biosynthesis \\
\hline GRP78 & ER & Chaperone \\
\hline hnRNP-M, a isoform 12 & Nucleus & RNA metabolism \\
\hline Hsp60 & Mitochondria & Chaperone \\
\hline NRAP isoform C & Cell membrane & Cellular structure \\
\hline Orp150 & ER & Chaperone \\
\hline Polyubiquitin & Cytoplasm & Protein degradation \\
\hline Ubiquinol-cytochrome-c reductase & Mitochondria & Metabolic enzyme \\
\hline UPP1 & Cytoplasm & Metabolic enzyme \\
\hline \multicolumn{3}{|c|}{ Proteins Whose Abundance Levels Were Significantly Decreased during COM Crystal Adhesion } \\
\hline Annexin A8 isoform 1 & Cell membrane & Signal transduction \\
\hline Annexin Al & Cell membrane & Signal transduction \\
\hline ATP synthase alpha subunit precursor & Mitochondria & ATP synthesis \\
\hline Beta tubulin & Microtubule & Cellular structure \\
\hline Centractin (Actin-related protein) & Associated with centrosome, cytosol & Cellular structure \\
\hline CG1550-PA & Cytoplasm & Metabolic enzyme \\
\hline CK8 & Intermediate filament & Cellular structure \\
\hline Cyclin A1 & Nucleus & Cell cycle regulator \\
\hline $\mathrm{EF}-1$, delta isoform 3 & Cytoplasm & Protein biosynthesis \\
\hline eIF-5A1 & Cytoplasm & Protein biosynthesis \\
\hline Enolase 1 & Cytoplasm & Metabolic enzyme \\
\hline PPIA & Cytoplasm & Protein biosynthesis \\
\hline PRDX3 & Mitochondrial membrane & Antioxidant enzyme \\
\hline Proteasome alpha 1 subunit & Cytoplasm & Protein degradation \\
\hline Proteasome subunit p42 & Cytoplasm & Protein degradation \\
\hline RhoGDI alpha & Cytoplasm & Inhibitor of Rho protein degradation \\
\hline TAGLN2 & Cytoplasm & Cellular structure \\
\hline TIM isoform 5 & Cytoplasm & Metabolic enzyme \\
\hline
\end{tabular}

${ }^{a}$ Three altered proteins: hypothetical protein (spot no. 15), unnamed protein product (spot no. 41), and unidentified protein (spot no. 19), of which function remains unknown are not included.

\section{Results}

Approximately 3-million MDCK cells were inoculated in each culture flask and maintained for $24 \mathrm{~h}$. A total of 10 semiconfluent flasks were then divided into two groups ( $n=5$ per group) and the culture medium was replaced by either COMcontaining (with $1000 \mu \mathrm{g} / \mathrm{mL}$ COM crystals) or COM-free medium. The cells were grown further for $48 \mathrm{~h}$. Crystal adhesion on the cell surface was clearly demonstrated using phase-contrast microscopy (Figure 1A) and SEM (Figure 1B). These crystals adhered tightly onto the cells as they remained adherent even after several washes with PBS. Interestingly, the borders of these crystals became blurred or interrupted, which indicated cell-crystal interactions. In a parallel experiment, time-course cell death assay using annexin V/propidium iodide double staining showed that \% cell death was gradually increased in MDCK cells exposed to COM crystals. At 48 h, \% cell death was significantly greater in the COM-exposed MDCK cells compared to the control MDCK cells (28.04 \pm 0.89 vs 16.71 $\pm 1.89 \%, p<0.001$ ) (Figure 2).

After the culture with or without COM crystals for $48 \mathrm{~h}$, MDCK cells were harvested from individual flasks and cellular proteins from each flask were extracted and resolved in each 2 -D gel ( $n=5$ gels per group). The resolved proteins were then visualized by SYPRO Ruby fluorescence stain and the visualized spots were analyzed by Image Master 2D Platinum (GE Healthcare) software, as detailed in Materials and Methods. From approximately 1200-1400 spots visualized in each 2-D gel, quantitative intensity analysis and statistics revealed significant changes in abundance levels of totally 53 protein spots in the COM-exposed cells (Figure 3). These altered proteins were then identified by Q-TOF MS (peptide mass fingerprinting; PMF) and/or MS/MS (peptide sequencing). Table 1 summarizes 


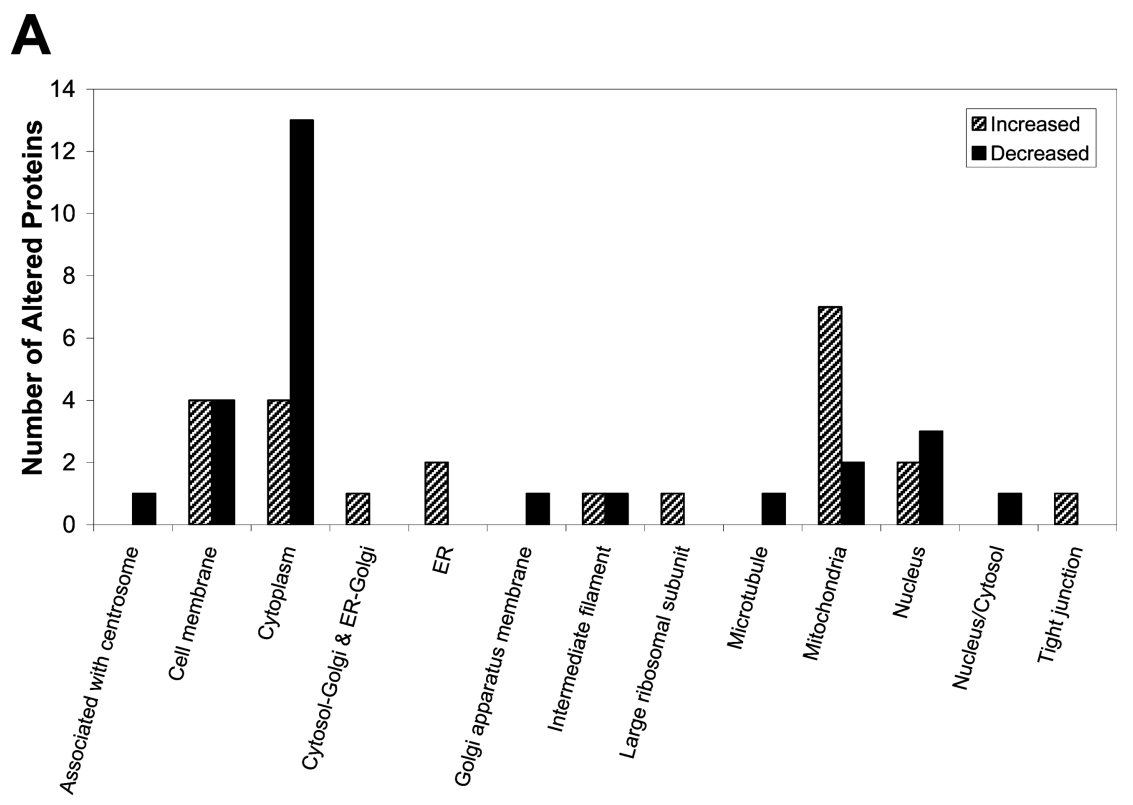

B

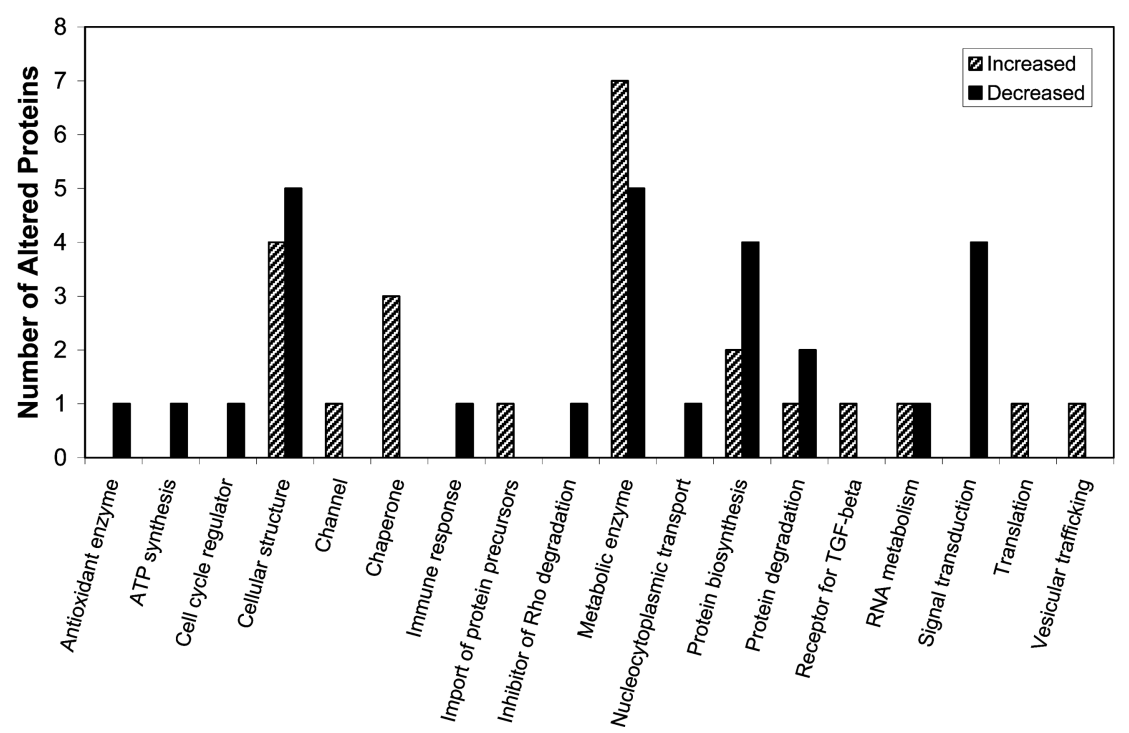

Figure 5. Numbers of the altered proteins in MDCK cells treated with COM crystals $(1000 \mu \mathrm{g} / \mathrm{mL})$ with respect to individual subcellular compartments $(\mathrm{A})$ and functional categories $(\mathrm{B})$.

identities, identification scores and other information related to protein identification and changes in their abundance levels. 2-D Western blot analysis was performed on randomly selected altered proteins to confirm the proteomic data. Figure 4 illustrates that the decreased levels in ezrin, enolase 1, and annexin Al (Anx I) were clearly confirmed by 2-D Western blot analysis.

In addition, bioinformatic analysis using the Swiss-Prot protein knowledgebase and TrEMBL computer-annotated supplement to Swiss-Prot (http://www.expasy.org/sprot/) was performed to obtain additional information (specifically, subcellular localization and functional significance) of the altered proteins; (Table 2). Numbers of the altered proteins, with respect to individual subcellular compartments and functional categories, are summarized in Figure 5, panels A and B, respectively.

\section{Discussion}

We demonstrated that COM crystals could adhere tightly to MDCK cells with subsequent detrimental effects to the cells (Figures 1 and 2). Exposure to COM crystals for $48 \mathrm{~h}$ (which might be considered as an acute phase response in vivo) caused increased cell death and alterations in abundance levels of several cellular proteins (25 proteins were increased, whereas other 28 were decreased). These data clearly indicate that COM crystals are toxic to distal renal tubular cells. Table 2 provides functional insights and subcellular localizations of these altered proteins. Regarding function, proteins involved in metabolic enzymatic pathways were mostly affected ( 7 proteins were increased, whereas 5 were decreased) (Figure 5B). The second and third most common functional groups affected by COMinduced toxicity included those involved in cell structure and cytoskeletal assembly (4 proteins were increased, whereas 5 
were decreased) and those involved in protein biosynthesis (2 proteins were increased, whereas 4 were decreased), respectively (Figure 5B). Regarding subcellular localization, the most affected compartment was cytoplasm (4 proteins were increased, whereas 13 were decreased) (Figure 5A). Mitochondria (7 proteins were increased, whereas 2 were decreased) and cell membrane (4 proteins were increased, whereas 4 were decreased) were the second and third most common compartments, respectively, affected by COM-induced toxicity (Figure 5A). Our data on subcellular compartments of renal tubular cells that were frequently affected by COM crystal-induced toxicity were consistent with those reported in other previous studies. ${ }^{12,20,21}$ The functional significance and potential roles of some of these altered proteins in the renal tubular toxicity induced by COM crystals are highlighted as follows.

Three chaperones (GRP78, Orp150 and Hsp60) had increased levels in MDCK cells after the exposure to COM crystals. GRP78 (or immunoglobulin-binding protein, BiP) is an ER luminal chaperone that can be activated by unfolded/misfolded proteins in the ER. ${ }^{22}$ Orp150, another ER chaperone, belongs to Hsp70 family and can be triggered by oxygen deprivation or hypoxia. ${ }^{23}$ While GRP78 and Orp150 are ER chaperones, Hsp60 (or mitochondrial matrix protein $\mathrm{P} 1$ ) is a mitochondrial chaperone that can prevent misfolding of proteins and promote refolding and proper assembly of unfolded/misfolded proteins in the mitochondrial matrix. ${ }^{24}$ Numerous studies have demonstrated an important role of these chaperones in defense mechanism against cellular toxicity and apoptosis. ${ }^{23,25-31}$ Our present study identified increased levels of these three chaperones in response to COM crystal adhesion, suggesting that their increases might be for protecting further cellular damage induced by COM crystals. However, the time-course cell death study implicated that their increases were not sufficient to protect COM crystal-induced cellular injury as the cell death was progressively increased when the exposure time was prolonged (Figure 2) and there were too many detrimental changes in cellular proteins that counterbalanced and finally overcame the protecting effects of these three chaperones (Table 2 and Figure 5B).

COM crystals caused several detrimental effects in MDCK cells. In particular, four proteins involved in protein biosynthesis [glutaminyl-tRNA synthetase (GlnRS) isoform 9, elongation factor 1 (EF-1) delta isoform 3, eukaryotic translational initiator factor 5A-1 (eIF-5A1), and peptidylprolyl isomerase A (PPIA)], a protein involved in ATP synthesis (ATP synthase alpha subunit precursor), and a cellular cycle regulator (cyclin A1) were significantly decreased after the exposure to COM crystals. These detrimental changes might clearly explain the reason that the three chaperones with increased levels (GRP78, Orp150 and Hsp60) could not completely protect the cells from further injury or apoptosis/necrosis induced by COM crystal adhesion.

Moreover, several proteins involved in cellular structure [beta tubulin, centractin, cytokeratin 8 (CK8), ezrin isoform 5 , and transgelin 2 (TAGLN2)] were also decreased. While tubulin ${ }^{32,33}$ and centractin ${ }^{34,35}$ are very important for cell cycle and division, ezrin $^{36-38}$ and transgelin $2^{39,40}$ are essential for other several cellular functions. The decreases in these proteins involved in cellular structure can definitely reduce cell integrity and also induce cellular vulnerability to stimuli or toxicants. Ezrin (also known as p81, cytovillin and villin-2) is a PDZ-domain phosphoprotein, belongs to an ezrin/radixin/moesin (ERM) family, and is a linker protein for the association between plasma membrane and actin cytoskeleton assembly. ${ }^{36}$ These ERM proteins govern cellular function through the Rho GTPase pathway. ${ }^{41,42}$ Ezrin also participates in other signal transduction pathways. ${ }^{37,38}$ Additionally, our data also showed the coordinated changes in several proteins involved in signal transduction, including RhoGDI alpha, meningioma expressed antigen 6 (Mgea6), annexin Al (Anx I), annexin A8 (Anx VIII) isoform 1 , and phosphatidyl ethanolamine binding protein 1 (PEBP1), all of which were also decreased. All these decreases were likely to worsen the cellular toxicity induced by COM crystals.

While several proteins involved in cellular structure and cytoskeletal assembly were decreased, some of these proteins on the other hand were increased. The mechanism underlying these disparate results remains unclear. Interestingly, most of these increased cellular structure proteins were plasma membrane [nebulin-related anchoring protein (NRAP) isoform C, and stomatin-like protein 2 (SLP-2) isoform 1] and tight junction (cingulin isoform CRA_b) proteins. Perhaps, these proteins might play important role in facilitating crystal binding or serve as adhesion molecules to bind with COM crystals. However, further investigation is required to confirm our hypothesis.

Interestingly, one antioxidative protein [peroxiredoxin 3 (PRDX3)] was significantly decreased during COM crystal adhesion. PRDX3 is a mitochondrial protein involved in the redox pathway and can protect cells from oxidative damage. Its reduction can decline the threshold of cells to the oxidative stress. ${ }^{43}$ This finding was consistent with the results reported in previous studies, which indicated that COM can induce oxidative damage to renal tubular epithelial cells. ${ }^{44-46}$ The decrease in PRDX3 might explain tubular oxidative injury induced by COM crystals.

It should be noted that we evaluated changes in the cellular proteome in MDCK cells after 48-h exposure to a high dose $(1000 \mu \mathrm{g} / \mathrm{mL})$ of COM crystals. Although we successfully identified several proteins that were altered in this setting, evaluation of changes in the cellular proteome induced by lower doses of COM crystals and at earlier time-points would lead to an understanding of "early responses" in distal renal tubular cells during cell-crystal interactions. Moreover, the study of proteome changes in proximal renal tubular cells would provide more insights into the molecular mechanisms of COM crystal-induced tubulotoxicity.

In summary, we successfully identified a set of proteins in MDCK cells that were altered by COM crystal-induced toxicity. Alterations in several of these proteins could explain the mechanistic pathways of COM crystal-induced toxicity in renal tubular epithelial cells. However, the functional significance of some altered proteins remains unclear and should be further elucidated to better understand the detrimental changes and adaptive responses in these cells during COM crystal adhesion, and to unravel the pathogenic mechanisms of kidney stone disease.

Abbreviations: 2-DE, two-dimensional electrophoresis; ACN, acetonitrile; Ak2, adenylate kinase isoenzyme 2; Anx I, annexin Al; Anx VIII, annexin A8; AST, aspartate aminotransferase; $\operatorname{BCAT}(\mathrm{m})$, branched-chain-amino-acid aminotransferase; CDC2, cell division cycle 2; CHAPS, 3-[(3-cholamidopropyl) dimethylamino]-1-propanesulfonate; CHCA, cyano-4-hydroxycinnamic acid; CK1, casein kinase 1; CK 7, cytokeratin 7; CK 8, cytokeratin 8; COM, calcium oxalate monohydrate; DTT, dithiothreitol; EDTA, ethylene diamine tetraacetic acid; EF-1, elongation factor 1; EF-Tu, Tu translation elongation factor; Eif2c2, eukaryotic 
translation initiation factor C2, 2 protein; eIF-5A1, eukaryotic translation initiation factor 5A-1; FBS, fetal bovine serum; GalNAG, alpha- $N$-acetylgalactosaminide; GlnRS, glutaminyltRNA synthetase; GRP78, $78 \mathrm{kDa}$ glucose-regulated protein; hnRNP-H3, heterogeneous nuclear ribonucleoprotein H3; hnRNP-M, heterogeneous nuclear ribonucleoprotein $\mathrm{M}$ isoform; Hsp60, $60 \mathrm{kDa}$ heat shock protein; IEF, isoelectric focusing; MDCK, Madin-Darby Canine Kidney; MEM, Eagle's minimum essential medium; Mgea6, meningioma expressed antigen 6; MS, mass spectrometry; MS/MS, tandem mass spectrometry; MVP, major vault protein; NRAP, nebulin-related anchoring protein; Orp150, $150 \mathrm{kDa}$ oxygen-regulated protein precursor; PEBP1, phosphatidylethanolamine binding protein 1; PIBF1, progesterone-induced blocking factor 1; PMF, peptide mass fingerprinting; PPIA, peptidylprolyl isomerase A; PRDX3, peroxiredoxin 3; Q-TOF, quadrupole time-of-flight; RhoGDI alpha, Rho GDP dissociation inhibitor alpha; RPL12, ribosomal protein L12; SEM, scanning electron microscopy; SLP-2, stomatin-like protein 2; TAGLN2, transgelin 2; TFA, trifluoroacetic acid; TGFR1, TGF beta receptor type I; TIM, triosephosphate isomerase; TOMM40, translocase of outer membrane $40 \mathrm{kDa}$ subunit; UPP1, uridine phosphorylase 1; UPR, unfolded protein response.

Acknowledgment. This study was supported by The Thailand Research Fund, Commission on Higher Education, Mahidol University, the National Research Council of Thailand, Siriraj Grant for Research and Development, and the National Center for Genetic Engineering and Biotechnology (to V. Thongboonkerd), and by Siriraj Graduate Thesis Scholarship (to T. Semangoen). We are also grateful to the Core Facilities for Proteomics and Structural Biology Research, Institute of Biological Chemistry, Academia Sinica, Taiwan.

\section{References}

(1) Coe, F. L.; Evan, A.; Worcester, E. Kidney stone disease. J. Clin. Invest. 2005, 115, 2598-2608.

(2) Amato, M.; Lusini, M. L.; Nelli, F. Epidemiology of nephrolithiasis today. Urol. Int. 2004, 72 Suppl 1, 1-5.

(3) Schubert, G. Stone analysis. Urol. Res. 2006, 34, 146-150.

(4) Sheng, X.; Ward, M. D.; Wesson, J. A. Crystal surface adhesion explains the pathological activity of calcium oxalate hydrates in kidney stone formation. J. Am. Soc. Nephrol. 2005, 16, 1904-1908.

(5) Rabinovich, Y. I.; Esayanur, M.; Daosukho, S.; Byer, K. J.; El Shall, H. E.; Khan, S. R. Adhesion force between calcium oxalate monohydrate crystal and kidney epithelial cells and possible relevance for kidney stone formation. J. Colloid Interface Sci. 2006, 300, 131-140.

(6) Khan, S. R. Crystal-induced inflammation of the kidneys: results from human studies, animal models, and tissue-culture studies. Clin. Exp. Nephrol. 2004, 8, 75-88.

(7) Khan, S. R.; Byer, K. J.; Thamilselvan, S.; Hackett, R. L.; McCormack, W. T.; Benson, N. A.; Vaughn, K. L.; Erdos, G. W. Crystal-cell interaction and apoptosis in oxalate-associated injury of renal epithelial cells. J. Am. Soc. Nephrol. 1999, 10 Suppl 14, S457-S463.

(8) Asselman, M.; Verhulst, A.; De Broe, M. E.; Verkoelen, C. F. Calcium oxalate crystal adherence to hyaluronan-, osteopontin-, and CD44expressing injured/regenerating tubular epithelial cells in rat kidneys. J. Am. Soc. Nephrol. 2003, 14, 3155-3166.

(9) Muthukumar, A.; Selvam, R. Renal injury mediated calcium oxalate nephrolithiasis: role of lipid peroxidation. Renal Failure 1997, 19, 401-408.

(10) Habibzadegah-Tari, P.; Byer, K.; Khan, S. R. Oxalate induced expression of monocyte chemoattractant protein-1 (MCP-1) in HK-2 cells involves reactive oxygen species. Urol. Res. 2005, 33, 440-447.

(11) Thamilselvan, S.; Khan, S. R.; Menon, M. Oxalate and calcium oxalate mediated free radical toxicity in renal epithelial cells: effect of antioxidants. Urol. Res. 2003, 31, 3-9.
(12) McMartin, K. E.; Wallace, K. B. Calcium oxalate monohydrate, a metabolite of ethylene glycol, is toxic for rat renal mitochondrial function. Toxicol. Sci. 2005, 84, 195-200.

(13) Khand, F. D.; Gordge, M. P.; Robertson, W. G.; Noronha-Dutra, A. A.; Hothersall, J. S. Mitochondrial superoxide production during oxalate-mediated oxidative stress in renal epithelial cells. Free Radical Biol. Med. 2002, 32, 1339-1350.

(14) Thongboonkerd, V. Proteomics in nephrology: current status and future directions. Am. J. Nephrol. 2004, 24, 360-378.

(15) Thongboonkerd, V.; Malasit, P. Renal and urinary proteomics: Current applications and challenges. Proteomics 2005, 5, 10331042.

(16) Thongboonkerd, V. Proteomic analysis of renal diseases: Unraveling the pathophysiology and biomarker discovery. Expert Rev. Proteomics 2005, 2, 349-366.

(17) Saier, M. H., Jr. Growth and differentiated properties of a kidney epithelial cell line (MDCK). Am. J. Physiol. 1981, 240, C106-C109.

(18) Tsujihata, M. Mechanism of calcium oxalate renal stone formation and renal tubular cell injury. Int. J. Urol. 2008, 15, 115-120.

(19) Thongboonkerd, V.; Semangoen, T.; Chutipongtanate, S. Factors determining types and morphologies of calcium oxalate crystals: Molar concentrations, buffering, $\mathrm{pH}$, stirring and temperature. Clin. Chim. Acta 2006, 367, 120-131.

(20) Jonassen, J. A.; Cao, L. C.; Honeyman, T.; Scheid, C. R. Intracellular events in the initiation of calcium oxalate stones. Nephron Exp. Nephrol. 2004, 98, e61-e64.

(21) Meimaridou, E.; Jacobson, J.; Seddon, A. M.; Noronha-Dutra, A. A.; Robertson, W. G.; Hothersall, J. S. Crystal and microparticle effects on MDCK cell superoxide production: oxalate-specific mitochondrial membrane potential changes. Free Radical Biol. Med. 2005, 38, 1553-1564.

(22) Li, J.; Lee, A. S. Stress induction of GRP78/BiP and its role in cancer. Curr. Mol. Med. 2006, 6, 45-54.

(23) Ozawa, K.; Kuwabara, K.; Tamatani, M.; Takatsuji, K.; Tsukamoto, Y.; Kaneda, S.; Yanagi, H.; Stern, D. M.; Eguchi, Y.; Tsujimoto, Y.; Ogawa, S.; Tohyama, M. 150-kDa oxygen-regulated protein (ORP150) suppresses hypoxia-induced apoptotic cell death. J. Biol. Chem. 1999, 274, 6397-6404.

(24) Bukau, B.; Horwich, A. L. The Hsp70 and Hsp60 chaperone machines. Cell 1998, 92, 351-366.

(25) Miyake, H.; Hara, I.; Arakawa, S.; Kamidono, S. Stress protein GRP78 prevents apoptosis induced by calcium ionophore, ionomycin, but not by glycosylation inhibitor, tunicamycin, in human prostate cancer cells. J. Cell Biochem. 2000, 77, 396-408.

(26) Yu, Z.; Luo, H.; Fu, W.; Mattson, M. P. The endoplasmic reticulum stress-responsive protein GRP78 protects neurons against excitotoxicity and apoptosis: suppression of oxidative stress and stabilization of calcium homeostasis. Exp. Neurol. 1999, 155, 302-314.

(27) Bando, Y.; Tsukamoto, Y.; Katayama, T.; Ozawa, K.; Kitao, Y.; Hori, O.; Stern, D. M.; Yamauchi, A.; Ogawa, S. ORP150/HSP12A protects renal tubular epithelium from ischemia-induced cell death. FASEB J. 2004, 18, 1401-1403.

(28) Kitao, Y.; Ozawa, K.; Miyazaki, M.; Tamatani, M.; Kobayashi, T.; Yanagi, H.; Okabe, M.; Ikawa, M.; Yamashima, T.; Stern, D. M.; Hori, O.; Ogawa, S. Expression of the endoplasmic reticulum molecular chaperone (ORP150) rescues hippocampal neurons from glutamate toxicity. J. Clin. Invest. 2001, 108, 1439-1450.

(29) Ozawa, K.; Kondo, T.; Hori, O.; Kitao, Y.; Stern, D. M.; Eisenmenger, W.; Ogawa, S.; Ohshima, T. Expression of the oxygen-regulated protein ORP150 accelerates wound healing by modulating intracellular VEGF transport. J. Clin. Invest. 2001, 108, 41-50.

(30) Gupta, S.; Knowlton, A. A. HSP60, Bax, apoptosis and the heart. J. Cell Mol. Med. 2005, 9, 51-58.

(31) Ghosh, J. C.; Dohi, T.; Kang, B. H.; Altieri, D. C. Hsp60 regulation of tumor cell apoptosis. J. Biol. Chem. 2008, 283, 5188-5194.

(32) Verhey, K. J.; Gaertig, J. The tubulin code. Cell Cycle 2007, 6, 21522160.

(33) Pellegrini, F.; Budman, D. R. Review: tubulin function, action of antitubulin drugs, and new drug development. Cancer Invest. 2005, 23, 264-273.

(34) Clark, S. W.; Meyer, D. I. Centractin is an actin homologue associated with the centrosome. Nature 1992, 359, 246-250.

(35) Clark, I. B.; Meyer, D. I. Overexpression of normal and mutant Arplalpha (centractin) differentially affects microtubule organization during mitosis and interphase. J. Cell Sci. 1999, 112 (Pt 20), 3507-3518.

(36) Algrain, M.; Turunen, O.; Vaheri, A.; Louvard, D.; Arpin, M. Ezrin contains cytoskeleton and membrane binding domains accounting for its proposed role as a membrane-cytoskeletal linker. J. Cell Biol. 1993, 120, 129-139. 
(37) Tsukita, S.; Yonemura, S. ERM (ezrin/radixin/moesin) family: from cytoskeleton to signal transduction. Curr. Opin. Cell Biol. 1997, 9, 70-75.

(38) Naba, A.; Reverdy, C.; Louvard, D.; Arpin, M. Spatial recruitment and activation of the Fes kinase by ezrin promotes HGF-induced cell scattering. EMBO J. 2008, 27, 38-50.

(39) Yu, H.; Konigshoff, M.; Jayachandran, A.; Handley, D.; Seeger, W.; Kaminski, N.; Eickelberg, O. Transgelin is a direct target of TGF\{beta\}/Smad3-dependent epithelial cell migration in lung fibrosis. FASEB J. 2008, 22, 1778-1789.

(40) Shapland, C.; Hsuan, J. J.; Totty, N. F.; Lawson, D. Purification and properties of transgelin: a transformation and shape change sensitive actin-gelling protein. J. Cell Biol. 1993, 121, 1065-1073.

(41) Mackay, D. J.; Esch, F.; Furthmayr, H.; Hall, A. Rho- and racdependent assembly of focal adhesion complexes and actin filaments in permeabilized fibroblasts: an essential role for ezrin/ radixin/moesin proteins. J. Cell Biol. 1997, 138, 927-938.

(42) D’Angelo, R.; Aresta, S.; Blangy, A.; Del Maestro, L.; Louvard, D.; Arpin, M. Interaction of ezrin with the novel guanine nucleotide exchange factor PLEKHG6 promotes RhoG-dependent apical cytoskeleton rearrangements in epithelial cells. Mol. Biol. Cell 2007, 18, 4780-4793.

(43) Mukhopadhyay, S. S.; Leung, K. S.; Hicks, M. J.; Hastings, P. J.; Youssoufian, H.; Plon, S. E. Defective mitochondrial peroxiredoxin-3 results in sensitivity to oxidative stress in Fanconi anemia. J. Cell Biol. 2006, 175, 225-235.

(44) Meimaridou, E.; Lobos, E.; Hothersall, J. S. Renal oxidative vulnerability due to changes in mitochondrial-glutathione and energy homeostasis in a rat model of calcium oxalate urolithiasis. Am. J. Physiol.: Renal Physiol. 2006, 291, F731-F740.

(45) Patel, A. B.; Robertson, W. G.; Choong, S.; Hothersall, J. S. Heatshock protein 25 ameliorates calcium oxalate crystal-mediated oxidative stress in renal epithelial cells. BJU Int. 2006, 98, 10941099.

(46) Byer, K.; Khan, S. R. Citrate provides protection against oxalate and calcium oxalate crystal induced oxidative damage to renal epithelium. J. Urol. 2005, 173, 640-646.

PR8002408 